\title{
Optimal Power Flow Using Genetic Algorithm
}

\author{
Fernando Buzzulini Prioste \\ Dept. of Electrical Engineering \\ Santa Catarina State University \\ Joinville, Brazil \\ fernando.prioste@udesc.br
}

\begin{abstract}
This paper presents a genetic algorithm (GA) to solve Optimal Power Flow (OPF) problems, optimizing electricity generation fuel cost. The GA based OPF is a derivative free optimization technique that relies on the evaluation of several points in the parameter search space strictly on the objective function. A 3 bus system and the IEEE 30 bus test system are used to validate the developed GA based OPF by means of comparisons with an interior point based optimal power flow.
\end{abstract}

Index Terms-Electric Power Systems, Power Flow, Optimal Power Flow, Nonlinear Optimization, Genetic Algorithm, Interior Point

\section{INTRODUCTION}

Since the emergence of electric power systems, there has always been a search for increasingly efficient methods to solve the power flow problem. The power flow consists of solving a set of nonlinear algebraic equations with quadratic and trigonometric terms. When its solution is found, state variables of a power system are available and several information about the system may also be used, for example, to system planning and stability studies. Among the most successful algorithms applied on the power flow solution are those that use the Newton-Raphson method, proposed by Van Ess and Griffin [4]. This method underwent improvements in [5], who introduced matrix techniques using sparsity as a means of implementing the Newton-Raphson method for large networks. Early developments of digital power flow calculations were reviewed by Tinney and Powell [6]. Currently there are several books and computer programs that explain and solve the power flow problem [7]-[9], [19].

Prior to the development of algorithms for the power flow solution, there was already a concern with increasing the operational efficiency of electrical power systems [1], since the costs, mainly of fossil fuels, increased the price of tariffs of electricity. In order to optimize operating costs, in [2] the economic dispatch of generating units was presented. The economic dispatch problem, as it takes only generation costs, was extended by Carpentier [3], in order to take into account not only the generation costs, but also the losses in the transmission systems. That's where the term optimal power flow [10], [12]-[14] arose and since then, the use of new techniques for solution have been proposed over the years

The author thanks FAPESC (Fundação de Apoio à Pesquisa e Inovação do Estado de Santa Catarina).
[15], [16], [21]. The Alternating Current Optimal Power Flow (ACOPF) is at the heart of Independent System Operator (ISO) power markets, and is solved for system planning, day-ahead markets and even at every 5 minutes. Even nowadays, 60 years after the problem was formulated, there are not a fast and robust solution technique available for its full solution. Depending on the amount of electric energy production of a country, finding a good solution technique for the full ACOPF could save tens of billions of dollars annually. The OPF problem is a large, highly nonlinear, multimodal, constrained optimization problem that may have nonconvex, nonsmooth, and nondifferentiable objective functions, in which there may be more than one optimal local solution.

The methods or techniques that have been implemented to optimize the OPF are divided into two categories: deterministic methods and evolutionary methods. Deterministic methods include linear, nonlinear programming, quadratic programming and interior point method [17], [18]. These methods may have problems in handling many local minima because of the nonconvexity of OPF problems. Due to limitations of deterministic methods, evolutionary methods such as genetic algorithm (GA) and particle swarm optimization have been proposed for solving the OPF as alternative [24], [26]-[29]. Genetic algorithms offer a powerful approach to these optimization problems, because their global search techniques are more likely to converge toward the global solution. Also GA, simultaneously, evaluates several points in the parameter space strictly on the objective function and do not need to assume that the search space is differentiable or continuous.

This work aims to develop an OPF algorithm whose solution is based on the use of Genetic Algorithm. The development of such a program takes into account the fact that writing nonlinear optimization programs with restrictions applied to electrical power systems is a cumbersome process, mainly due its dimensions. Also, the use of evolutionary algorithms in OPF solution is not such a widespread idea in Brazil if compared to deterministic methods. To validate the developed program, Matpower [19], which uses interior point method to solve OPF will be used. Two test systems are used for this.

The document is structured as follows: in Section II, the problem formulation is presented. In this Section the conventional power flow and the optimal power flow are described. In Section II-C, a general ideia of the developed Genetic Algorithm Optimal Power Flow (GAOPF) is presented. In 
Section III, the 3-bus and 30-bus test systems are presented together with the obtained results, which validates the developed GAOPF program. Finally, comments and conclusions are presented.

\section{PROBlem FORMUlation}

In this section, the basic idea and formulation of the power flow and optimal power flow are presented. Also the basic idea of how to implement a Genetic Algorithm based Power Flow is explained.

\section{A. Basic Power Flow Problem}

The load flow or power flow problem consists of finding the steady-state operating point of an electric power system. The the aim is to obtain all bus voltages and complex power flowing through all network components such as generating units, transmission lines and loads. The power flow is the most widely used application in electric power systems, both in operating and in planning environments, either as a standalone tool or as a subroutine within more complex processes such as stability analysis, optimization problems, etc. It can be formulated as a set of nonlinear algebraic equality or inequality constraints. These constraints represent both Kirchhoff's laws and network operation limits.

$$
\begin{aligned}
P_{i}(V, \theta)=P_{G i}-P_{D i} \quad \forall i=1, m \\
Q_{i}(V, \theta)=Q_{G i}-Q_{D i} \quad \forall i=1, m \\
P_{G i_{\text {min }}} \leq P_{G i}(V, \theta) \leq P_{G i_{\max }} \quad \forall i=1, N_{G} \\
Q_{G i_{\text {min }}} \leq Q_{G i}(V, \theta) \leq Q_{G i_{\max }} \quad \forall i=1, N_{G} \\
V_{i_{\text {min }}} \leq V_{i} \leq V_{i_{\max }} \quad \forall i=1, m
\end{aligned}
$$

where

$P_{G i}$ : is the generator real power output at to bus $i$;

$Q_{G i}$ : is the generator reactive power output at to bus $i$;

$P_{D i}$ : is the real load power at bus $i$;

$Q_{D i}$ : is the reactive load power at bus $i$;

$P_{i}$ : is the real net power injection at bus $i$;

$Q_{i}$ : is the reactive net power injection at bus $i$;

$V_{i}$ : is the voltage magnitude at bus $i$;

$\theta_{i}$ : is the voltage angle at bus $i$;

$N_{G}$ is the number of generators on the system;

$m$ is the number of power system buses.

Also (1a) and (1b) are the power flow equations represented, respectively, as follows:

$$
\begin{aligned}
& P_{i}(V, \theta)=V_{i} \sum_{j=1}^{m} V_{j}\left(G_{i j} \cos \theta_{i j}+B_{i j} \sin \theta_{i j}\right) \\
& Q_{i}(V, \theta)=V_{i} \sum_{j=1}^{m} V_{j}\left(G_{i j} \sin \theta_{i j}-B_{i j} \cos \theta_{i j}\right)
\end{aligned}
$$

where [8], [9]:

$G_{i j}$ : is the series conductance between buses $i j$;

$B_{i j}$ is the series susceptance between buses $i j$;

The inequalities (1c) - (1d) reflect the limits on physical devices and (1e) the limits created to ensure system security.
Each bus provides two equations and four unknowns, which means that two variables per bus must be specified to solve the resulting load flow equations. Depending on which variables are specified, three main types of buses can be distinguished: Load or PQ buses: Both active $P_{D_{i}}^{s p}$ and reactive $Q_{D_{i}}^{s p}$ power absorbed by the sum of loads connected at the bus are specified. It must be verified if the difference between the specified power and the evaluated power through equations is null. This leads to the following bus constraints:

$$
P_{i}=P_{i}^{s p}=-P_{D_{i}}^{s p} ; \quad Q_{i}=Q_{i}^{s p}=-Q_{D_{i}}^{s p} .
$$

leaving the phasor voltage components, $\dot{V}_{i}=V_{i} \angle \theta_{i}$, as the remaining unknowns.

Generation or PV buses or also voltage controlled bus: These are buses where the voltage regulator of a local generator keeps the voltage magnitude to a specified value $\left(V_{s p_{i}}\right)$. Furthermore, the active power $P_{G_{i}}^{s p}$ injected by the generator is specified. This specification may be according to certain economic criteria. Taking into account the possible local load demand, the resulting net constraints are:

$$
P_{i}=P_{i}^{s p}=P_{G i}^{s p}-P_{D_{i}}^{s p} ; \quad V_{i}=V_{i}^{s p} .
$$

leaving $Q_{i}$ and $\theta_{i}$ as unknowns. A particular case of PV bus arises when a reactive power compensator (rotating or static), equipped with voltage regulator, is connected to a bus. In such case, $P_{G i}^{s p}=0$.

If only those two types of buses were considered, all injected complex powers should be specified a priori, which requires that active and reactive losses be known in advance. However, power losses depend on the resulting power flows and cannot be accurately determined until the load flow itself is solved. Therefore, the complex power of at least a generator should be left as an unknown. For convenience, the voltage phasor of the generating bus whose complex power remains unspecified is taken as reference for phase angles. This particular PV bus, is the third type of bus and it is known as the slack or swing bus. Such bus is usually chosen among those generating buses with largest capacity, frequently being in charge of frequency regulation duties. For the slack bus, the complex voltage is fully specified whereas both power components, active and reactive, belong to the set of unknowns. Thus, the solution of the set of nonlinear algebraic equations (1a) - (1e), is only possible if there exists a slack bus. On the slack bus the generation level is calculated so as to meet the active and reactive power balance of entire power system using (5), with $S_{i}=P_{i} \pm j Q_{i}$ :

$$
S_{G_{e_{\text {Slack }}}}=\sum_{j=1}^{m} S_{D_{j}}+\sum_{j=1}^{m} S_{\text {loss }_{j}}-\sum_{j=1}^{N G-1} S_{G_{e n_{j}}}
$$

Such condition, if attended, assures that the power system operates at its nominal frequency $60 \mathrm{~Hz}$ or $50 \mathrm{~Hz}$. The slack bus concept is a mathematical artifact, without any direct link to the real world, as no bus in the system is explicitly in charge of balancing (5). 
In order to solve the power flow problem, several iterative methods where employed through the years, but one of the most effective is the Newton-Raphson method [5], [7], [8]. This method successively improves the unknown values through first-order approximations of the set of nonlinear algebraic equations. For initial guess values $x_{0}$, close to the solution, also known as flat start, i.e., $\dot{V}_{i}=V_{i} \angle \theta_{i}=1,0 \angle 0 \mathrm{pu}$, the Newton-Raphson method converges quadratically. Irrespective of the network dimension, starting from the flat voltage profile, takes from three to five iterations to attain convergence [11].

\section{B. Optimal Power Flow Problem}

An OPF is an optimization problem applied to power flow, which determines the optimal settings of the power system variables in a way that the equality and inequality constraints are satisfied [3], [10], [12], [14]. OPF objective function and constraints can be formulated as (6a) - (6f)

$$
\begin{aligned}
& \min \quad F=\sum_{i=1}^{N_{G}} f_{i}\left(P_{G i}\right) \\
& \text { s.t.: } \quad P_{i}(V, \theta)=P_{G i}-P_{D i} \quad \forall i=1, m \\
& Q_{i}(V, \theta)=Q_{G i}-Q_{D i} \quad \forall i=1, m \\
& P_{G i_{\text {min }}} \leq P_{G i}(V, \theta) \leq P_{G i_{\max }} \quad \forall i=1, N_{G} \\
& Q_{G i_{\text {min }}} \leq Q_{G i}(V, \theta) \leq Q_{G i_{\max }} \quad \forall i=1, N_{G} \\
& V_{i_{\text {min }}} \leq V_{i} \leq V_{i_{\text {max }}} \quad \forall i=1, m
\end{aligned}
$$

where equality and inequality constraints, functions and variables are the same defined in the Basic Power Flow Problem, Section II-A. There are several kinds of objective functions, among the most common are: Generation cost objective function, Active power loss objective function, Pollutant Gases Emission objective function and combinations of the mentioned ones in order to obtain multi-objective functions [29].

In this work, the generator cost function was chosen to minimize the total energy production cost of the power system. This kind of objective function for the OPF reflects the costs associated with generating power in the system. The quadratic cost model for thermal power generation will be used and is defined in (7), $f_{i}\left(P_{G i}\right)$ represents the active power generation cost by each generator and $a_{i}, b_{i}$ and $c_{i}$, the $i^{\text {th }}$ cost function coefficients of the quadratic cost model at each thermal generator.

$$
f_{i}\left(P_{G i}\right)=a_{i}+b_{i} P_{G i}+c_{i} P_{G i}^{2}
$$

Such objective function (6a), where $N_{G}$ is the total number of generation units, is used to minimize the total system costs associated with active power generation $P_{G_{i}}$, and does not necessarily minimize the costs for a particular area within the power system.

As a nonlinear optimization problem, several solution methods have been proposed through the years [5], [12], [14]-[18]. In order to solve the classical OPF problem, in this work the Matlab Toolbox Matpower [19] was used for comparison purposes. Matpower has interior point method for nonlinear optimization [18], [20], [21] as default algorithm for the OPF solution.

\section{Genetic Algorithm Optimal Power Flow Problem}

The Genetic Algorithms (GAs) are part of the evolutionary algorithms family which in turn are inspired in the Nature. Such algorithms are powerful stochastic search tools based on natural selection and genetics [22], [23]. They offer a powerful approach to nonlinear constrained optimization problems solutions such as ACOPF and have found extensive applications in solving global optimization searching problems. Their advantage relies on the evaluation of many points in the parameter space and such search space does not need to be differentiable or continuous as methods in [17], [18].

To solve ACOPF with a simple GA, some details must be addressed: First it is necessary to generate an initial binary string (chromosome) which associates a binary code to each active power $\left(P_{G i}\right)$ for all the system generators. An initial population is created with several $\left(P_{G i} \in P_{G_{i m i n}}<\right.$ $\left.P_{G i}<P_{G_{\text {imax }}}\right)$ and these chromosomes originate the initial search space or population. Then the initial population passes through the processes of reproduction, crossover, mutation and immigration so that new intermediate populations are created.

In the reproduction process, the chromosomes (or possible combinations of solutions $P_{G i}$ ) are evaluated in the objective function and the best chromosomes are selected. These selected chromosomes (sets of $P_{G i}$ ) then pass to the crossover process where segments of their bits are exchanged with each other to generate their offspring. The chromosomes with larger scores have more chances to be mixed with other ones.

Mutation is the secondary operator and prevents the premature stopping of the algorithm in a local solution. The mutation operator is defined by a random bit value change in a chosen string (chromosome) with a low probability of such change. The mutation adds a random search character to the genetic algorithm, and it is necessary to avoid that, after some generations, all possible solutions become very similar ones [23], [25].

Another procedure which may be used in GA is the process of immigration [30]. This process raises the fitness of the population as a whole and enriches its diversity, i.e., random chromosomes are created and added to the population in order to improve its diversity and the possibility of finding a better minima.

After these procedures, the resulting chromosomes $\left(P_{G i}\right)$ that belong to the next Generation/population are tested on the objective function. If this is the optimum Generation, the best/optimal chromosome $\left(P_{G i}^{*}\right)$ is chosen and the process is stopped as the optimum solution is found, otherwise it is necessary to repeat the steps above. In Fig. 1 there is GA-Based Optimization Approach Flowchart. Finally, if is necessary to know all the active and reactive power flowing through the system, a conventional power flow must be performed. All the simulations by GAOPF on this work have used the Matlab $g a(x)$ function as well all as the following control parameters:

- Population size: 50

- Number of generations: 300

- Crossover probability: 0.8 - Matlab default 


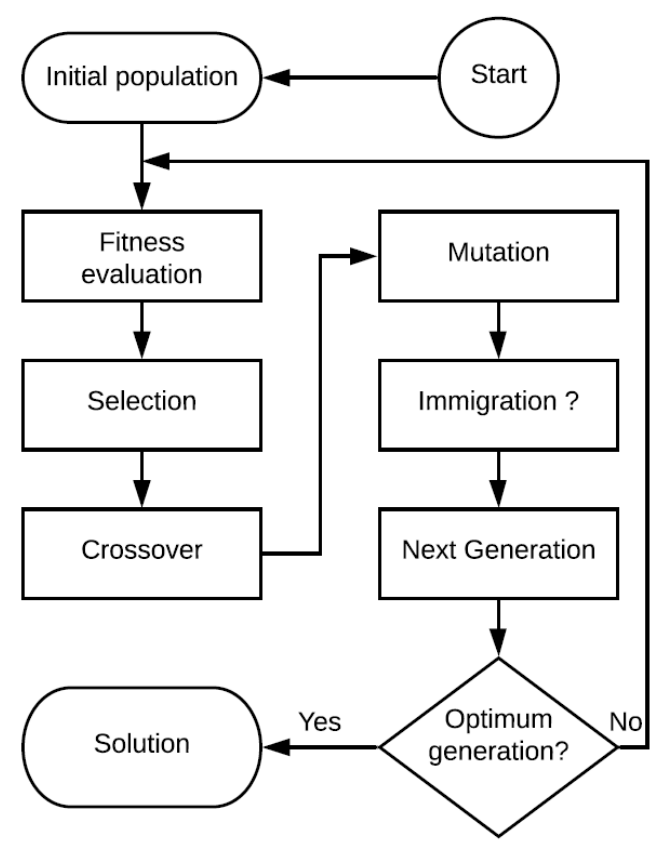

Fig. 1. GA-Based Optimization Approach Flowchart.

- Mutation probability: 0.01 - Matlab default

\section{Test Systems}

In this section two test systems are presented in order to validate the developed GAOPF. The developed algorithm objective is to optimize the active power dispatch in order to reduce the cost of electricity production. The first test system has 3 bus and is a fictitious electric power system, the second one is the IEEE 30 bus system [12]. Results of the developed GAOPF and Matpower [19] are compared. Matpower is a software whose main purpose is to solve optimal power flows, using by default, the interior point method for that.

\section{A. 3 Bus System}

The 3 Bus test system is the same one presented in [9]. It is a fictitious system, composed by 2 synchronous generators, 3 branches and a single load located at bus 2. Bus 1 is the slack bus, the PV (voltage controlled bus) is bus 3 and the PQ bus is bus 2. The one-line diagram is given in Fig. 2 .

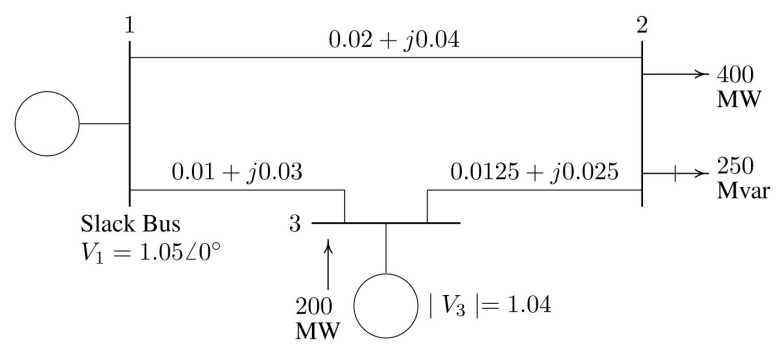

Fig. 2. 3 Bus Test System.
Generators data and Cost coefficients are given on Table I. According to the presented nomenclature, Section II-B, the nonlinear optimization problem (6a)-(6f), has $N_{G}=2, m=3$ and the objective function is a sum of two distinct second order polynomials given by (7).

The prime objectives of using the 3 Bus system is to compare results of an optimal load-flow solution, which gives the optimal active and reactive power dispatch for a static power-system loading condition, with the ones obtained by the developed Genetic Algorithm Optimal Power Flow. This comparison is made in order to validate the developed GAOPF, besides understanding some important aspects of the OPF problem. The validation of the GAOPF was done comparing some crucial results with the ones obtained by the Matpower [19].

TABLE I

3 Bus System Generator Data

\begin{tabular}{|c|c|c|c|c|c|c|}
\hline Bus & $P_{G_{\min }}$ & $P_{G_{\max }}$ & $Q_{G_{\min }-\max }$ & \multicolumn{3}{|c|}{ Cost coefficients * } \\
No. & MW & MW & MVAr & a & b & c \\
\hline 1 & 50 & 300 & -200 to 200 & 0.0 & 2 & 0.00375 \\
\hline 3 & 50 & 250 & -200 to 200 & 0.0 & 1.75 & 0.0175 \\
\hline
\end{tabular}
$f_{i}\left(P_{G i}\right)=a_{i}+b_{i} P_{G i}+c_{i} P_{G i}^{2}$ in $\$ / \mathrm{hr}$.

The results of OPF and GAOPF are summarized in Table II and Table III. Both optimization algorithms led to almost the same results in active power generation as can be observed in Table II. It is possible to note that $P_{G_{1}}$ achieved its maximum allowed capacity, limited by the $P_{G 1_{\max }}$ bound, highlighted in bold, when the using NLOPF. On the other way, $P_{G_{1}} \approx$ $P_{G 1_{\max }}$ when GAOPF was performed. Despite that, in both situations, the necessary condition imposed by (5) was satisfied [8], [9].

TABLE II

Power Generation - 3 Bus System

\begin{tabular}{|c|c|c|c|c|}
\hline $\begin{array}{c}\text { Optimization } \\
\text { Method }\end{array}$ & $\begin{array}{c}G_{1} \\
\text { MW }\end{array}$ & $\begin{array}{c}G_{3} \\
\text { MW }\end{array}$ & $\begin{array}{c}G_{1} \\
\text { MVAr }\end{array}$ & $\begin{array}{c}G_{3} \\
\text { MVAr }\end{array}$ \\
\hline NLP-OPF & $\mathbf{3 0 0 . 0 0}$ & 119.47 & 90.06 & 199.79 \\
\hline GA-OPF & 299.14 & 120.35 & 112.95 & 176.94 \\
\hline
\end{tabular}

Also the voltage magnitudes of the 3 Bus system have almost the same profile, as seen on Table III. It is important to note that a slight difference between $\left|\dot{V}_{3}\right|$ voltages in OPF and GAOPF implies in a quite significant change on the reactive power generated by $G_{1}$ and $G_{3}$, Table II. In both cases the imposed bus voltage inequality constraints are $0.95 \mathrm{pu}<V_{m}<1.05 \mathrm{pu}$ and there were not violations at the end of the optimization processes.

Recalling (5) is possible to verify the active and reactive power system losses $\left(S_{\text {loss }}\right)$. Such power losses agree with the ones presented on Table IV. Also it is easy to see why the production cost $(\$ / h r)$ calculated by the GAOPF is bigger than the one calculated by NLOPF. The $c$ cost coefficient, Table I, of $c_{G_{3}} \approx 4,66 c_{G_{1}}$ and it is proportional to $P_{G 3}^{2}$, Table II. 
TABLE III

3 Bus System Bus Voltages

\begin{tabular}{|c|c|c|c|}
\hline $\begin{array}{c}\text { Optimization } \\
\text { Method }\end{array}$ & $\begin{array}{c}\left|\dot{V}_{1}\right| \\
\mathrm{pu}\end{array}$ & $\begin{array}{c}\left|\dot{V}_{2}\right| \\
\mathrm{pu}\end{array}$ & $\begin{array}{c}\left|\dot{V}_{3}\right| \\
\text { pur }\end{array}$ \\
\hline NLP-OPF & 1.05 & 0.975 & 1.044 \\
\hline GA-OPF & 1.05 & 0.972 & 1.040 \\
\hline
\end{tabular}

TABLE IV

3 Bus System Production Cost and Losses

\begin{tabular}{|c|c|c|c|}
\hline $\begin{array}{c}\text { Optimization } \\
\text { Method }\end{array}$ & $\begin{array}{c}\text { Total Cost } \\
\$ / \mathrm{hr}\end{array}$ & $\begin{array}{c}P_{\text {Losses }} \\
\mathrm{MW}\end{array}$ & $\begin{array}{c}Q_{\text {Losses }} \\
\text { MVAr }\end{array}$ \\
\hline NLP-OPF & 1396.00 & 19.47 & 39.84 \\
\hline GA-OPF & 1397.90 & 19.46 & 39.88 \\
\hline
\end{tabular}

\section{B. IEEE 30 Bus System}

An adaptation of the IEEE 30-bus standard test-system, Fig. 3, is used to compare the Matpower [19] OPF solution with the developed GAOPF algorithm in Matlab. The data used to represent the 30 Bus System may be found in [12]. The objective function to be minimised is again the total system active-power generation cost, allowing all the generator $\left(P_{G i}\right) \mathrm{MW}$-outputs to be controllable and considered as one of the inequality constraints. The IEEE 30 Bus System has 6 generators, 41 branches and its total amount of load is $S_{D}=283.4+j 126.2$ MVA. Observing the nonlinear optimization problem, set of equations (6a)- (6f), with $N_{G}=6$ and $m=30$ it is possible to determine the problem dimension and envision the complexity that may arise when applying nonlinear optimization methods in a real electric power system such as the Brazilian Interconnected Power System (BIPS).

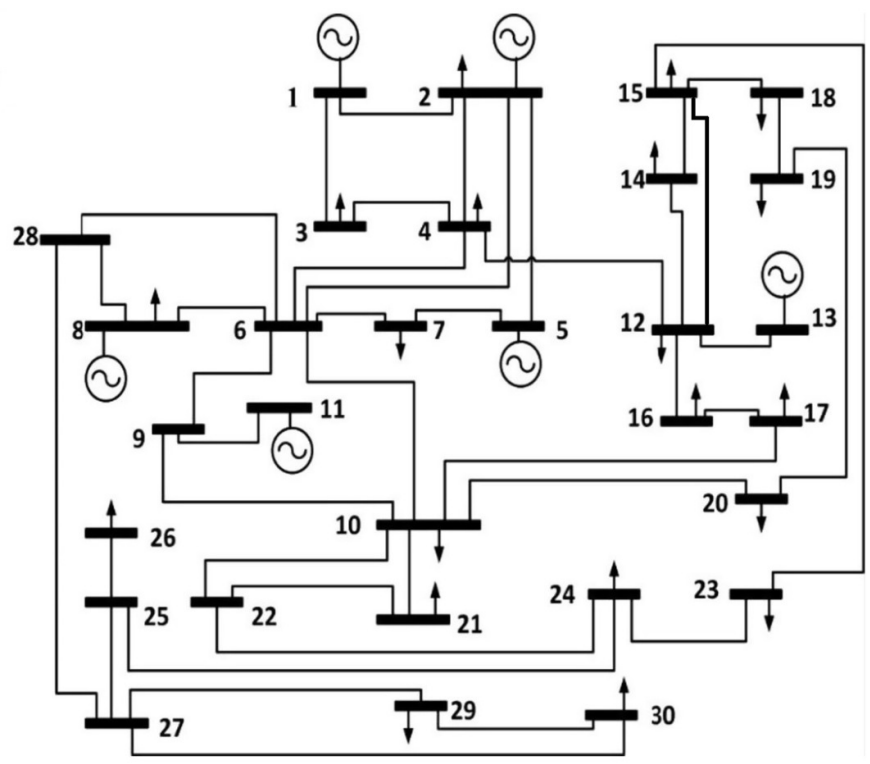

Fig. 3. IEEE 30 Bus Power System.

The 30 Bus System generator data are presented on Table V. All the complex power $\left(S_{G i}\right)$ limits are presented as the respective Cost Coefficients to be used. These data are used on inequality constraints (6d), (6e) and also on the objective function (6a), respectively.

TABLE V

IEEE 30 BUS SYSTEM GENERATOR DATA

\begin{tabular}{|c|c|c|c|c|c|c|}
\hline Bus & $P_{G_{\min }}$ & $P_{G_{\max }}$ & $Q_{G_{\min }-\max }$ & \multicolumn{3}{|c|}{ Cost coefficients ${ }^{*}$} \\
No. & MW & MW & MVAr & a & b & c \\
\hline 1 & 50 & 200 & -20 to 250 & 0.0 & 2 & 0.02 \\
\hline 2 & 20 & 80 & -20 to 100 & 0.0 & 1.75 & 0.0175 \\
\hline 5 & 15 & 50 & -15 to 80 & 0.0 & 1.0 & 0.0625 \\
\hline 8 & 10 & 35 & -15 to 60 & 0.0 & 3.25 & 0.00834 \\
\hline 11 & 10 & 30 & -10 to 50 & 0.0 & 3.0 & 0.025 \\
\hline 13 & 12 & 40 & -15 to 60 & 0.0 & 3.0 & 0.025 \\
\hline
\end{tabular}

The optimal power flow was performed with Matpower (NLP-OPF) and also with the developed algorithm (GA-OPF). On Table VI it is possible to compare the optimal resulting active power generation by generator and method. The bold numbers in Table VI indicate that the maximum active power $\left(P_{G_{\max }}\right)$ was reached and limited by its respective constraints presented on Table V. Optimal MW-outputs achieved by both algorithms were quite similar. It can be observed that only $G_{1}$ and $G_{5}$ are far from their respective maximum active power limits, mainly because of their contribution to the total amount cost of energy production.

TABLE VI

Power Generation - IEEE 30 Bus

\begin{tabular}{|c|c|c|c|c|c|c|}
\hline $\begin{array}{c}\text { Optimization } \\
\text { Method }\end{array}$ & $\begin{array}{c}G_{1} \\
\text { MW }\end{array}$ & $\begin{array}{c}G_{2} \\
\text { MW }\end{array}$ & $\begin{array}{c}G_{5} \\
\text { MW }\end{array}$ & $\begin{array}{c}G_{8} \\
\text { MW }\end{array}$ & $\begin{array}{c}G_{11} \\
\text { MW }\end{array}$ & $\begin{array}{c}G_{13} \\
\text { MW }\end{array}$ \\
\hline NLP-OPF & 70.95 & $\mathbf{8 0 . 0 0}$ & 33.17 & $\mathbf{3 5 . 0 0}$ & $\mathbf{3 0 . 0 0}$ & 38.88 \\
\hline GA-OPF & 70.12 & $\mathbf{8 0 . 0 0}$ & 32.85 & $\mathbf{3 5 . 0 0}$ & $\mathbf{3 0 . 0 0}$ & $\mathbf{4 0 . 0 0}$ \\
\hline
\end{tabular}

The IEEE 30 Bus System voltage profiles, by bus number and algorithm, are presented in Fig. 4. The voltage profiles are very similar, which implies that the reactive power $\left(Q_{G_{i}}\right)$ injected by each generator, established by the optimization method, in the system also have close values. It is also important to mention that the voltage inequality constraints $\left(0.95 p u<V_{m}<1.085 p u\right)$ were not violated at the end of the optimization processes.

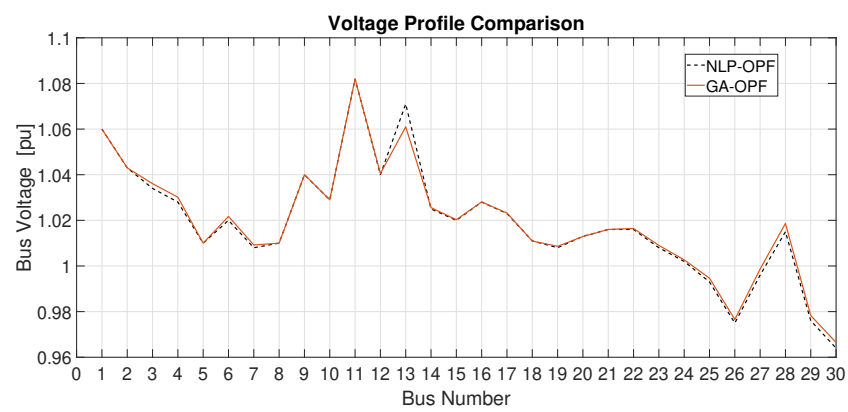

Fig. 4. Voltage Profile by Bus - IEEE 30 Bus System. 
As the active and reactive power generated and also the voltage profiles are very similar, it is expected that the total cost of energy production $\sum_{i=1}^{6} f_{i}\left(P_{G i}\right)$ on the objective function (6a) will also be. The minimum optimal energy production cost are shown on Table VII as well as active and reactive power losses through the 30 Bus System.

TABLE VII

IEEE 30 Bus System PRoduction Cost ANd Losses

\begin{tabular}{|c|c|c|c|}
\hline $\begin{array}{c}\text { Optimization } \\
\text { Method }\end{array}$ & $\begin{array}{c}\text { Total Cost } \\
\$ / \mathrm{hr}\end{array}$ & $\begin{array}{c}P_{\text {Losses }} \\
\text { MW }\end{array}$ & $\begin{array}{c}Q_{\text {Losses }} \\
\text { MVAr }\end{array}$ \\
\hline NLP-OPF & 987.37 & 4.59 & 22.42 \\
\hline GA-OPF & 986.63 & 4.51 & 22.46 \\
\hline
\end{tabular}

\section{CONCLUSIONS}

Results of the developed GA based OPF has been shown in this work. Solutions to the OPF problem has been applied and presented to a 3 bus and to the IEEE 30 bus electric power systems. The developed algorithm main objective is to optimize the active power dispatch. Results such as optimal fuel costs, complex generated power, voltage magnitudes as well systems losses were compared with the ones obtained by Matpower, which solves optimal power flows using an interior point method. Comparison between both algorithms results shown that all were quite similar. An advantage of GA over interior point optimization method is the fact that GA is derivative free and may be applied to nonconvex cost functions, besides the possibility of being coded to work on parallel computers. On the other way, the main disadvantage of GA is its stochastic nature, which means that not always the solution they provide to the OPF problem is guaranteed to be the optimum. As compared with the results obtained by the interior point method, which always finds the same deterministic solution, the GAOPF optimal solutions are always in a region that presents small deviations at the vicinity of the optimum solution points.

\section{ACKNOWLEDGMENT}

The author thanks FAPESC (Fundação de Apoio à Pesquisa e Inovação do Estado de Santa Catarina) for all support.

\section{REFERENCES}

[1] STAHL, E. C. M. Load division in interconnections. Electrical World, Brooklyn, p. 434-438, 1930.

[2] KIRCHMAYER, L. K. Economic operation of power systems. New York: Wiley, 1958.

[3] Carpentier, J. Contribution á l'étude du dispatching économique. Bulletin de la Societe Francaise des Electriciens, London, v. 3, n. 1, p. 431-447, 1962.

[4] Van Ess, J. E. and Griffin, J. H., "Elimination methods for load flow studies", AlEE Transactions, Vol. 80, pp. 299-304, 1961.

[5] W. F. Tinney and C. E. Hart, "Power Flow Solution by Newton's Method," in IEEE Transactions on Power Apparatus and Systems, vol. PAS-86, no. 11, pp. 1449-1460, Nov. 1967, doi: 10.1109/TPAS.1967.291823.

[6] Tinney, W. F. and Powell, W. L., "Notes on Newton-Raphson method for solution of AC power flow problem", IEEE Short Course, Power Systems Planning, 1971.

[7] G.W. Stagg (Autor), A.H. El-Abiad, "Computer Methods in Power System Analysis", McGraw-Hill Inc.,US - 1968
[8] J. Grainger and W. Stevenson, "Power System Analysis", McGraw-Hill Education; 1st edition, January 1, 1994.

[9] H. Saadat, "Power Systems Analysis", Mc Graw Hill; 2nd edition, 2002.

[10] H. W. Dommel and W. F. Tinney, "Optimal Power Flow Solutions," in IEEE Transactions on Power Apparatus and Systems, vol. PAS-87, no. 10, pp. 1866-1876, Oct. 1968, doi: 10.1109/TPAS.1968.292150.

[11] B. Stott, Effective starting process for Newton-Raphson load flows, IEE Proceedings, 118, 1971, 983-987.

[12] O. Alsac and B. Stott, "Optimal Load Flow with Steady-State Security," in IEEE Transactions on Power Apparatus and Systems, vol. PAS-93, no. 3, pp. 745-751, May 1974, doi: 10.1109/TPAS.1974.293972.

[13] H. H. Happ, "Optimal power dispatch: A comprehensive survey," in IEEE Transactions on Power Apparatus and Systems, vol. 96, no. 3, pp. 841-854, May 1977, doi: 10.1109/T-PAS.1977.32397.

[14] D. I. Sun, B. Ashley, B. Brewer, A. Hughes and W. F. Tinney, "Optimal Power Flow By Newton Approach," in IEEE Transactions on Power Apparatus and Systems, vol. PAS-103, no. 10, pp. 2864-2880, Oct. 1984, doi: 10.1109/TPAS.1984.318284

[15] A., Jr. Santos 1 and G.R.M. da Costa , "Optimal-power-flow solution by Newton's method applied to an augmented Lagrangian function" Source: Volume 142, Issue 1, January 1995, p. 33 - 36, doi 10.1049/ipgtd: 19951586

[16] Xihui Yan and V. H. Quintana, "Improving an interior-point-based OPF by dynamic adjustments of step sizes and tolerances," in IEEE Transactions on Power Systems, vol. 14, no. 2, pp. 709-717, May 1999, doi: $10.1109 / 59.761902$.

[17] J. A. Momoh, R. Adapa and M. E. El-Hawary, "A review of selected optimal power flow literature to 1993. I. Nonlinear and quadratic programming approaches," in IEEE Transactions on Power Systems, vol. 14, no. 1, pp. 96-104, Feb. 1999, doi: 10.1109/59.744492.

[18] J. A. Momoh, M. E. El-Hawary and R. Adapa, "A review of selected optimal power flow literature to 1993. II. Newton, linear programming and interior point methods," in IEEE Transactions on Power Systems, vol. 14, no. 1, pp. 105-111, Feb. 1999, doi: 10.1109/59.744495.

[19] R. D. Zimmerman, C. E. Murillo-Sanchez (2020). MATPOWER (Version 7.1) [Software]. Available: https://matpower.org

[20] Anders Forsgren, P. E. Gill, M. H. Wright, "Interior Methods for Nonlinear Optimization", SIAM REVIEW, Vol. 44, No. 4, pp. 525-597, 2006.

[21] Capitanescu, F.; Glavic, M.; Ernst, D.;Wehenkel, L., "Interior-point based algorithms for the solution of optimal power flow problems." Electr. Power Syst. Res. 2007, 77, 508-517.

[22] J. H. Holland, "Adaptation in natural and artificial systems". Ann Arbor, MI: The University of Michigan Press; 1975.

[23] D. E. Goldberg, "Genetic Algorithms in Search, Optimization and Machine Learning", Addison Wesley Publishing Company, Ind. USA, 1989.

[24] A. G. Bakirtzis, P. N. Biskas, C. E. Zoumas and V. Petridis, ”Optimal power flow by enhanced genetic algorithm," in IEEE Transactions on Power Systems, vol. 17, no. 2, pp. 229-236, May 2002, doi: 10.1109/TPWRS.2002.1007886.

[25] T. Bouktir, L. Slimani, M. Belkacemi, "A Genetic Algorithm for Solving the Optimal Power Flow Problem", Leonardo Journal of Sciences, Issue 4, January-June 2004

[26] D. Devaraj and B. Yegnanarayana, "Genetic-algorithm-based optimal power flow forsecurity enhancement", IEE Proc.-Gener. Transm. Distrib., Vol. 152, No. 6, November 2005

[27] J. Yuryevich and Kit Po Wong, "Evolutionary programming based optimal power flow algorithm," in IEEE Transactions on Power Systems, vol. 14 , no. 4, pp. 1245-1250, Nov. 1999, doi: 10.1109/59.801880.

[28] M.A. Abido, "Optimal power flow using particle swarm optimization", Electrical Power and Energy Systems 24, 2002

[29] S. Kahourzade, A. Mahmoudi, H. B. Mokhlis, "A comparative study of multi-objective optimal power flow based on particle swarm, evolutionary programming, and genetic algorithm, Electrical Engineering (Archiv fur Elektrotechnik) Volume 97 issue 12015.

[30] F. Ornelas, M. Meza, A. Padilla, F. Padilla, J. Ponce and A. Ochoa, "Genetic Algorithm with Immigration Like Strategies of Diversification," 2010 Ninth Mexican International Conference on Artificial Intelligence, 2010, pp. 11-15, doi: 10.1109/MICAI.2010.33. 